\title{
Granuloma anular maculoso
}

\section{Patch granuloma annulare}

Sofía C. Juárez ${ }^{1}$, Ana L. Gallmann², Julieta R. Brusa ${ }^{3}$, María N. Andrade ${ }^{4}$, Luciana Ragazzini ${ }^{5}$, María S. Gómez Zanni ${ }^{5}$, Anahí L. Bringas ${ }^{5}$, Virginia R. López Gamboa ${ }^{5}$, Andrés Guidi ${ }^{6}$ y Mariana B. Papa ${ }^{7}$

\section{RESUMEN}

El granuloma anular maculoso es una variante rara de granuloma anular, que representa un desafío diagnóstico. Su incidencia se desconoce y se presenta con mayor frecuencia en las mujeres de entre 40 y 70 años. Se asocia a las mismas entidades y responde a los mismos tratamientos que las otras variantes clínicas de granuloma anular.
Se presentan los casos de 5 mujeres con diagnóstico de granuloma anular maculoso, que recibieron diferentes tratamientos tópicos y sistémicos, con respuestas clínicas variables.

Palabras clave: granuloma anular, granuloma anular maculoso, enfermedad granulomatosa.

Dermatol. Argent. 2021, 27 (2): 53-58

\section{ABSTRACT}

Patch-type granuloma annulare is a rare variant of granuloma annulare, thus which represents diagnostic challenge. It has an unknown incidence and occurs most often in women between 40 and 70 years of age. It presents similar associations and responds to treatment as the clinical variants. We present 5 female patients with patch-type granuloma annulare, who received different topical and systemic treatments, with variable clinical responses.

Key words: granuloma annulare, patch-type granuloma annulare, granulomatous disease.

Dermatol. Argent. 2021, 27 (2): 53-58

Contacto de la autora: Sofía Carla Juárez

E-mail: juarez.sofiac@gmail.com

Fecha de trabajo recibido: $23 / 3 / 2021$

Fecha de trabajo aceptado: 11/6/2021

Conflicto de interés: los autores declaran que no existe conflicto de interés.

\section{INTRODUCCIÓN}

El granuloma anular (GA) es una dermatosis inflamatoria crónica benigna, de etiología desconocida y casi siempre autolimitada, cuya prevalencia real se ignora ${ }^{1-4}$. Existen variantes según la presentación clínica, entre las que se encuentra la forma maculosa, también llamada eritematosa, de tipo parche o en napa, descripta en 1932 por Monash ${ }^{4,5}$. Se presenta una serie de 5 casos de GA maculoso, escasamente comunicado en la bibliografía nacional e internacional, y su asociación a alteraciones metabólicas en la mayoría de las pacientes, que requirió un manejo interdisciplinario. 


\section{CASOS CLÍNICOS}

Los 5 casos corresponden a pacientes mujeres, con un rango de edad de 31 a 94 años, que presentaban múltiples antecedentes personales patológicos (Tabla 1). Las pacientes consultaron al servicio de Dermatología por máculas eritematovioláceas, de límites definidos, policíclicos, con un centro de lesión más claro, de diferentes tamaños, levemente pruriginosas y de distintos tiempos de evolución (entre 1 mes y 4 años). Las localizaciones más frecuentes fueron el tronco, el abdomen y los muslos (Fotos 1A, 1B, 1C, 1D). Una sola paciente (caso 1) consultó por una única lesión ubicada en la cara lateral derecha del tronco (Foto 1E). Se les realizaron biopsias cutáneas y estudios histopatológicos a todas las pacientes, así como estudios sanguíneos que incluyeron: hemograma completo, reactantes de fase aguda (eritrosedimentación, proteína $\mathrm{C}$ reactiva), prueba de tolerancia oral a la glucosa e insulinemia a los 0 y los 120 minutos, perfil lipídico, perfil tiroideo y pruebas serológicas virales para hepatitis $\mathrm{B}$ y $\mathrm{C}$, citomegalovirus, virus de la inmunodeficiencia humana (VIH) y virus de Epstein-Barr. Predominaron las alteraciones tiroideas y metabólicas en 3 de las 5 pacientes, que fueron derivadas a las respectivas especialidades

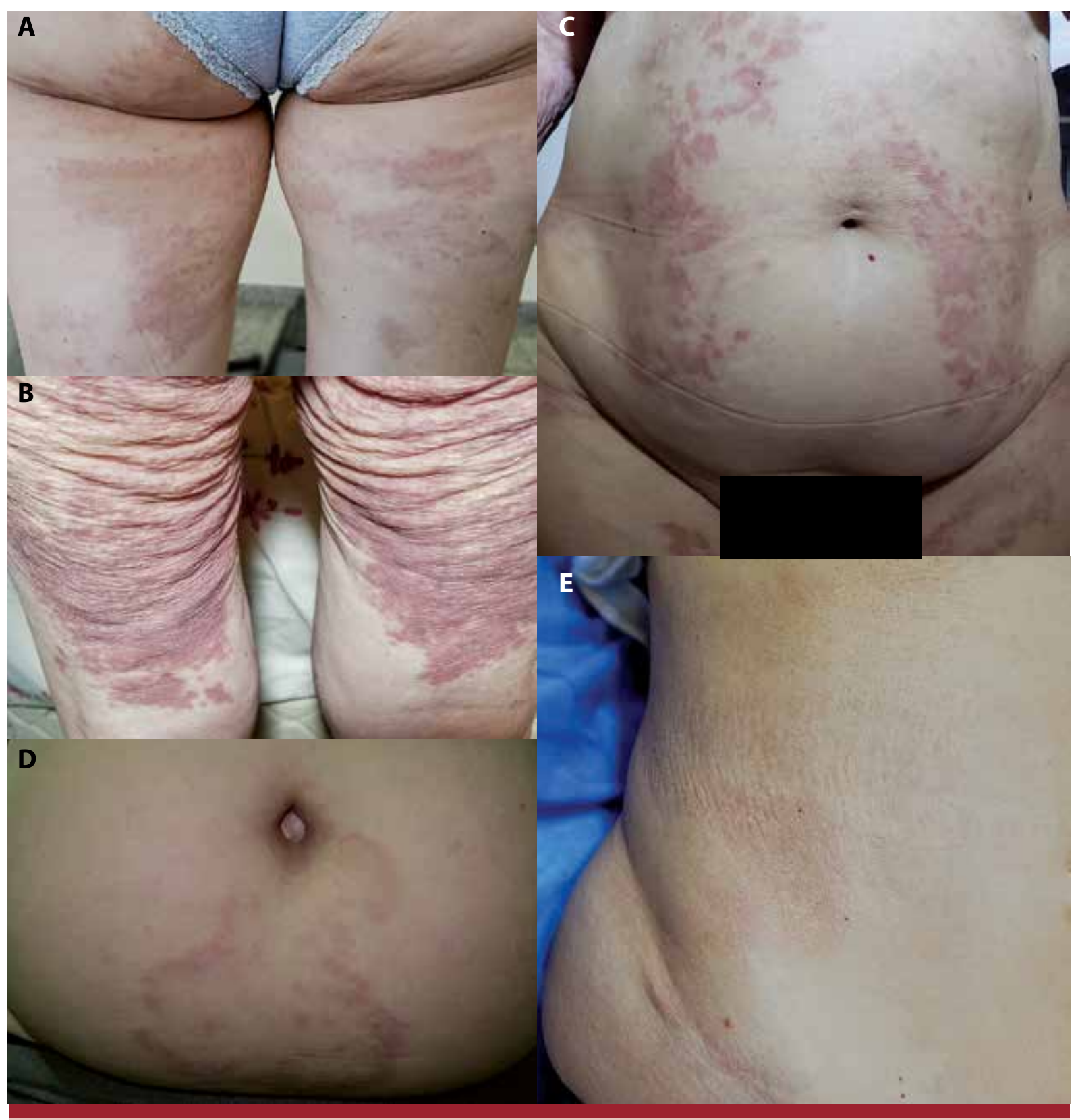

FOT0 1: Máculas eritematovioláceas de bordes policíclicos. A) Caso 2: lesiones en la región posterior de los muslos. B) Caso 3: lesiones en la región posterior de los muslos. C) Caso 4: lesiones en el abdomen. D) Caso 5: lesiones infraumbilicales. E) Caso 1: lesión en el flanco derecho. 


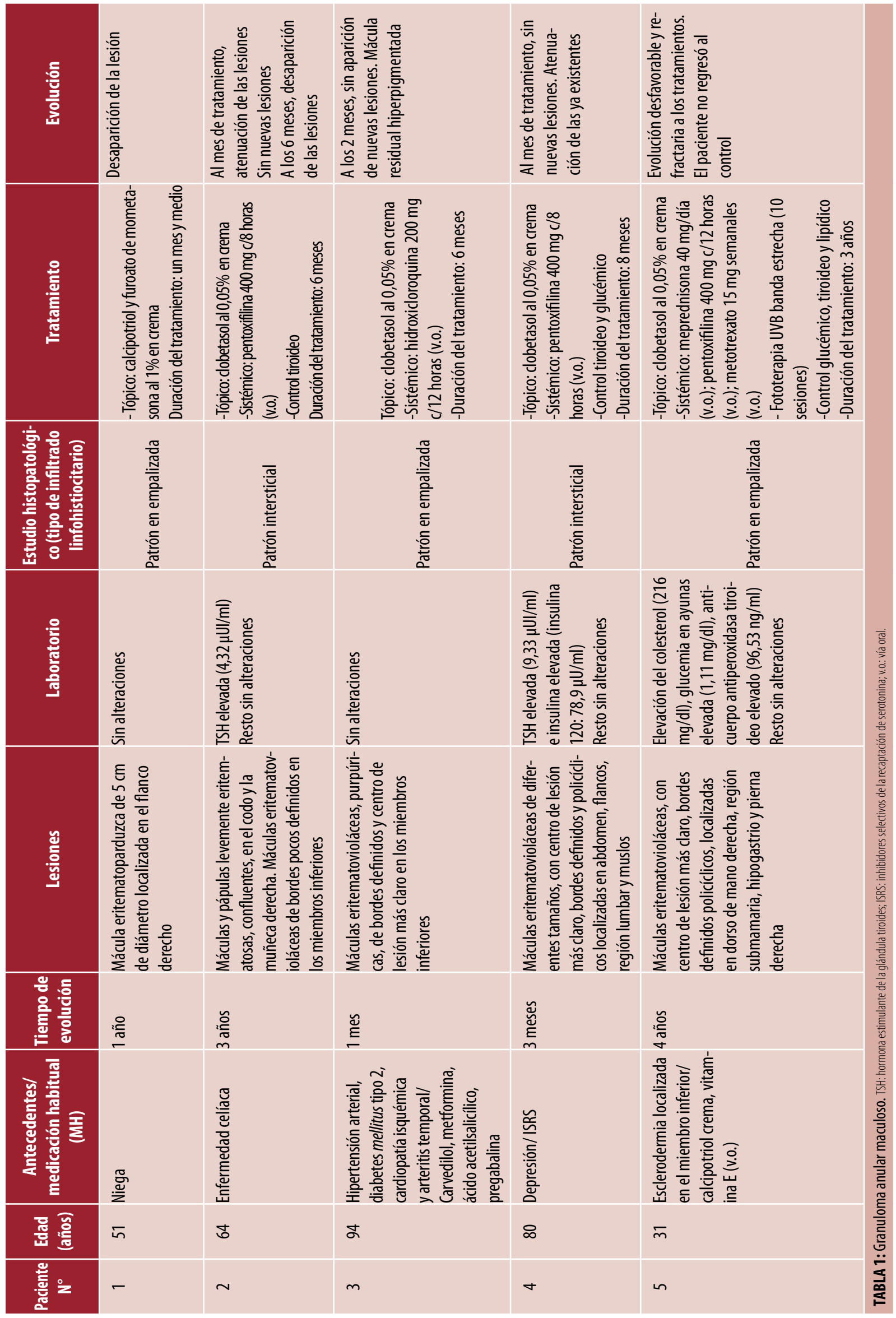


para su manejo en conjunto, mientras que las restantes 2 tuvieron resultados con parámetros normales, una de ellas con diabetes mellitus controlada. Ninguna presentó serología infecciosa positiva. Los resultados se muestran en la Tabla 1.

El estudio histopatológico con tinción de hematoxilina y eosina de las muestras cutáneas de todas las pacientes fue compatible con un granuloma anular. En tres muestras, se evidenció en la dermis un infiltrado linfocítico histiocitario en forma de empalizada difusa, con células pálidas gigantes multinucleadas. Las dos muestras restantes presentaron el patrón intersticial, con un infiltrado linfocítico histiocitario distribuido entre los haces de colágeno y con presencia de mucina (Fotos 2A y 2B).

En cuanto al tratamiento según cada caso, se realizó terapia tópica, sistémica o ambas. En el caso 1, por la presencia de una sola lesión, se optó por un tratamiento tópico. En cambio, en el resto de las pacientes con formas generalizadas de GA se combinaron medidas tópicas con medicación sistémica (véase Tabla 1).

En 4 pacientes, se evidenció la atenuación o la resolución de las lesiones alrededor de los 45 días de instaurada la terapéutica, sin reaparición durante el período de tratamiento y control de cada paciente, lo cual se detalla en la Tabla 1. En el caso 5, la evolución fue tórpida a pesar del tratamiento, con mejorías clínicas transitorias y múltiples recidivas en un período de 4 años. Durante el transcurso de su patología, llevó a término dos embarazos sin complicaciones, período en el cual presentó una llamativa mejoría del GA.

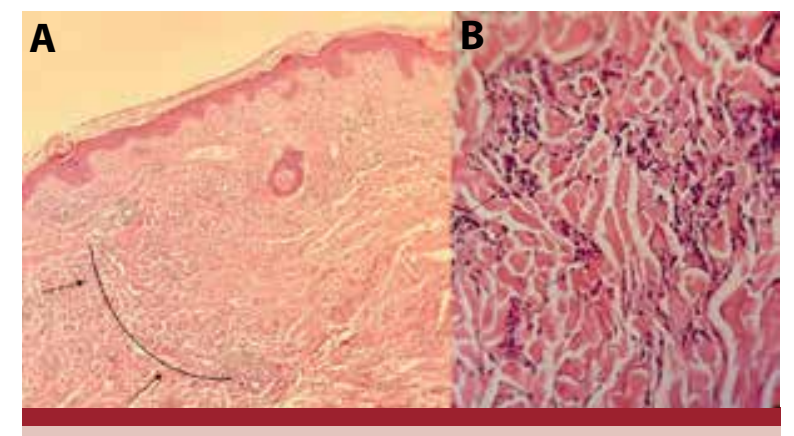

FOTO 2: Estudio histopatológico. A) Caso 3: histiocitos con patrón en empalizada en la dermis (HyE, 10X). B) Caso 1: histiocitos distribuidos intersticialmente entre los haces de colágeno (HyE, 40X).

\section{DISCUSIÓN}

El GA es una dermatosis inflamatoria crónica benigna, de etiología desconocida, casi siempre autolimitada, con resolución espontánea a los 2 años en la mayoría de los pacientes y que puede recurrir en un $40 \%$ de los $\operatorname{casos}^{1,2}$. Su prevalencia se estima en $0,1-0,4 \%$ y su distribución es mundial, sin predilección por ninguna etnia ${ }^{1-4,6}$. Es más frecuente en las mujeres, con una relación de 2:1 respecto de los hombres. Aparece a todas las edades, con predominio en los menores de 30 ańos ${ }^{1,3,7,8}$. La variante maculosa predomina en las mujeres, de preferencia entre los 40 y los 70 ańos, lo que coincide con las pacientes de esta serie 9 . No se han publicado datos sobre su prevalencia. Aunque se describieron casos familiares, la mayoría son de presentación esporádica. Se ha podido determinar la asociación del GA localizado con HLA-A2910 y del GA generalizado con HLA-A31 y HLA-Bw35 $5^{2,10}$. Las pacientes de los casos comunicados negaban familiares con esta afección y no se le realizó un estudio de HLA a ninguna de ellas.

Existen factores desencadenantes y enfermedades concomitantes como infección por VIH, hepatitis B y C, virus de Epstein-Barr, parvovirus B19, virus del herpes zóster, escabiosis, tuberculosis cutánea, picaduras de insectos, aplicación de vacunas (como la BCG, la antitetánica o la antidiftérica), exposición solar, fototerapia con psoraleno y radiación ultravioleta $A$ (PUVA) y mesoterapia. Se ha relacionado, además, con la ingesta de algunos fármacos, como el topiramato, los inhibidores del factor de necrosis tumoral, la nifedipina, la calcitonina y el alopurino ${ }^{2-4,8,9,11}$.

Se observó la asociación del GA con enfermedades metabólicas como diabetes mellitus, obesidad y dislipidemia, pero las publicaciones son contradictorias. Por su baja prevalencia, no se ha podido comprobar de manera fehaciente si su asociación a estas es real o producto del azar. Son necesarios datos epidemiológicos a mayor escala para dilucidar esta verdadera relación. Se cree que el estado inflamatorio crónico de estos pacientes podría estar relacionado con la aparición del GA. Existen también muchos informes de casos que comunican la asociación de GA con enfermedades tiroideas ${ }^{2,-8,810,12-14}$. En una serie de 23 casos de Khanna y North, publicada en 2020, la dislipidemia y la diabetes fueron las comorbilidades mayormente asociadas a GA maculoso ${ }^{10}$. En nuestra serie de casos, se encontraron alteraciones tiroideas en 3 pacientes, 2 presentaron alteraciones glucémicas y 1 presentó alteración del lipidograma. Todas fueron tratadas en conjunto con las especialidades correspondientes.

También, a pesar de la escasa evidencia bibliográfica, es necesario realizar un tamizaje de procesos malignos en los pacientes de edad avanzada, en aquellos con presentaciones atípicas o generalizadas y en los casos recalcitrantes de GA. Los tumores relacionados con mayor frecuencia son los linfomas de Hodgkin y no Hodgkin, como también el adenocarcinoma hepático, cervical uterino, de próstata o de mama; la micosis fungoide y leucemia mielomonocítica crónica ${ }^{2,6,9,11,14,15}$. 
La patogenia de esta enfermedad no se conoce. Se plantea que se trataría de una reacción inmunitaria de hipersensibilidad retardada de tipo IV a un antígeno desconocido. Se generaría una respuesta de tipo Th1 de linfocitos $\mathrm{CD}^{+}$, los cuales, mediante la liberación de interferón gamma y de otras citoquinas, estimularían la formación de un infiltrado monocitario granulomatoso en la dermis, con la liberación del factor de necrosis tumoral (TNF) alfa y de las metaloproteinasas 2 y 9 , que causan la degradación del tejido conjuntivo y un daño estructural en las fibras elásticas y de colágeno $^{2,4,5,10}$. Durante la gestación, puede haber mejoría clínica, como ocurrió con el caso 5 de nuestra serie de casos, ya que existe una disminución de la inmunidad mediada por los linfocitos Th1 y un aumento de la mediada por los Th2, necesaria para la tolerancia inmune placentaria $^{16}$.

Las manifestaciones cutáneas de GA son polimorfas, dado que hay diversas variantes clínicas: localizado, generalizado, perforante y subcutáneo o nodu$\operatorname{lar}^{4,9,10}$. Otras formas de presentación menos comunes son: en placa, de disposición lineal y maculoso ${ }^{4}$. Esta última variante macular es el subtipo diagnosticado con menor asiduidad ${ }^{3}$. Se presenta como máculas eritematosas, pardas o violáceas, no infiltradas, de forma redondeada u ovoidea, con configuración anular o sin ella, únicas o múltiples, de crecimiento centrífugo. Se localiza mayoritariamente en la región proximal de los miembros y rara vez compromete las palmas y las plantas ${ }^{17}$. Puede presentar o no descamación. En la mayoría de los casos, las lesiones son asintomáticas u ocasionan un prurito leve $\mathrm{e}^{3,9,11,18}$.

El diagnóstico se establece a partir de la sospecha clínica y las características dermatoscópicas e histopatológicas.

Mediante biopsia y tinciones con hematoxilina y eosina se evidencian, en la dermis superior y media, alteración focal del colágeno y las fibras elásticas, infiltrado linfohistiocítico y depósitos de mucina. Este último puede verse de forma más clara utilizando tinciones de hierro coloidal o azul alciano. Los histiocitos se pueden disponer en tres patrones: intersticial, en empalizada y granulomas epitelioides ${ }^{8,19,20}$.

La dermatoscopia del GA puede correlacionarse con los hallazgos histopatológicos. Es característica la visualización de vasos de morfología variable (punteados, lineales irregulares o ramificados) sobre un fondo rosado. Pueden observarse, además, áreas blanquecinas irregulares o globulares (correspondientes a fibrosis) y áreas amarillentas anaranjadas, focales o difusas, presentes en el patrón histopatológico en empalizada y ausentes en el patrón intersticial ${ }^{20}$.
Con respecto al GA maculoso, en la mayoría de los casos presenta un patrón intersticial, con una mínima degeneración de colágeno que no se organiza en nódulos. Las fibras elásticas aisladas aleatoriamente alternan con fibras normales, rodeadas de los histiocitos y los depósitos de mucina. Menos a menudo, se puede encontrar el patrón en empalizada, caracterizado por un núcleo central de colágeno degenerado, rodeado radialmente por el infiltrado de linfocitos, histiocitos y fibroblastos, fagocitosis de fibras elásticas por células gigantes en la periferia y depósitos de mucina ${ }^{2,3,4,9,11,17}$.

Ninguna prueba serológica es útil para el diagnóstico de GA. Los estudios de laboratorio para detectar patologías asociadas deben realizarse siempre que el interrogatorio, los antecedentes familiares, los signos y los síntomas sean sugestivos de sospecha.

Los diagnósticos diferenciales para considerar incluyen morfea temprana, parapsoriasis, micosis fungoide, eritema centrífugo anular, tiña, sarcoidosis macular, lepra, mucinosis y necrobiosis lipídica, ${ }^{9,18}$.

El tratamiento del GA maculoso es igual al del resto de los tipos de $\mathrm{GA}^{11,18}$. Debido al carácter benigno y autolimitado de esta dermatosis, la conducta expectante es una opción válida. Se describió su resolución posterior a la manipulación traumática, como la provocada por las biopsias, fenómeno conocido como iatrotropismo ${ }^{2,4,11,21}$. La elección del tratamiento se debe individualizar y basar fundamentalmente en la extensión de la enfermedad, la presencia o no de prurito y el grado de afectación psicológica del paciente ${ }^{4}$.

Para la forma localizada de la enfermedad, la terapia farmacológica de primera línea son los corticosteroides tópicos (clobetasol) o intralesionales (triamcinolona). Como segunda línea, se incluyen los inhibidores de la calcineurina (pimecrolimus, tacrolimus), el imiquimod, el interferón intralesional y la criocirugía ${ }^{8}$.

El tratamiento sistémico se reserva para las lesiones generalizadas. Sin embargo, no hay un tratamiento de primera línea establecido, ya que se cuenta con poca literatura especializada e insuficiente evidencia al respecto $^{9,11,22}$. Los corticosteroides (meprednisona) son una alternativa de uso frecuente ${ }^{21}$. La hidroxicloroquina, la isotretinoína o la dapsona son de elección por su seguridad y eficacia. Se describió el uso de otros fármacos como metotrexato, doxiciclina, vitamina $\mathrm{E}$ (uso oral y tópico), ésteres de ácido fumárico, alopurinol y pentoxifilina. Esta última se caracteriza por lograr, entre sus variados efectos, una modulación inmunitaria que disminuye la liberación del TNF por parte de los monocitos y la disminución de la respuesta leucocitaria ante las interleuquinas y el TNF. En nuestra experiencia, obtuvimos buenos resultados con esta última 
por su eficacia y escasos efectos adversos, motivo por el cual fue de elección en las pacientes 2,4 y 5 . Se puede comenzar con una dosis de $400 \mathrm{mg} /$ día y aumentar de manera progresiva hasta alcanzar un máximo de 1200 $\mathrm{mg} /$ día. Se comunicaron casos con la desaparición total de las lesiones después de 4 meses de tratamiento ${ }^{4}$.

Se describió también el tratamiento con pulsos mensuales de rifampicina, ofloxacina y minociclina (ROM) como una alternativa prometedora ${ }^{4}$. La terapia biológica con antagonistas del TNF-alfa (infliximab y adalimumab) se reserva para los casos recalcitrantes ${ }^{4,22}$.

Siempre que esté disponible, se puede optar, además, por el uso de fototerapia: terapia fotodinámica, UVB, UVA1 y PUVA. Esta última puede realizarse sola o en combinación con retinoides (RE-PUVA) ${ }^{4}$.

\section{BIBLIOGRAFÍA}

1. Domínguez López LMO, Aldama Caballero A, Rivelli V, Mendoza G. Granuloma anular: 17 casos con diferentes formas clínicas. Dermatología CMQ. 2007;5:195-198.

2. Navarro Hernández CA, Soto Ortiz JA, Solís Ledesma G, Navarro Jiménez BR. Granuloma anular: un reto dermatológico. Dermatol Rev Mex. 2018; 62:221-232.

3. Bollea Garlatti ML, Vacas AS, Valdivia D, Torre AC, et ál. Granuloma anular macular generalizado, asociado a linfoma Hodgkin. Dermatología CMQ 2015;13:318-322.

4. Corigliano M, Achenbach RE. Granuloma anular: un desafío diagnóstico y terapéutico. Rev Argent Dermatol. 2012;93.

5. Magnin PH, Palacios AM, Manzi R, Carmona JA, et ál. Granuloma anular gigante en napa, factor de error diagnóstico. Rev Argent Dermatol. 1990;71:1-6.

6. Schmieder SJ, Harper CD, Schmieder GJ. Granuloma anular. [Actualizado el 5 de enero de 2021]. En: StatPearls [Internet]. Treasure Island (FL): StatPearls Publishing; 2021 enero. Disponible en: https://www.ncbi.nlm.nih.gov/books/ NBK459377/> [Consulta: febrero 2021].

7. Rosenbach MA, Wanat KA, Reisenauer A, White KP, ét al. Granulomas no infecciosos. En: Bolognia JL, Schaffer JV, Cerroni L. Dermatología, 4. a ed., Elsevier, España, 2019:16501654.

8. Thornsberry LA, English JC. Etiology, diagnosis, and therapeutic management of granuloma annulare: an update. Am J Clin Dermatol. 2013;14:279-290.

9. Khanna U, North JP. Patch-type granuloma annulare: An institution-based study of 23 cases. J Cutan Pathol. 2020;47:785-793.

10. Alonso M, Martínez del Sel J, Allevato MAJ. Granuloma anular generalizado. Act Terap Dermatol. 2019;42:70-79.

11. Santos M, Fiesta L, Trila C, Abeldaño A. Granuloma anular
En conclusión, el GA implica un desafío diagnóstico por su polimorfismo lesional. La forma maculosa es una variante poco frecuente, de la que existen escasas publicaciones en la literatura médica nacional e internacional. Si bien la bibliografía es contradictoria en cuanto a su asociación con enfermedades sistémicas, en 3 de las pacientes detectamos alteraciones metabólicas que no eran conocidas y que debieron ser tratadas interdisciplinariamente, por lo que quizá sean necesarios más estudios para dilucidar esta relación. No hay una terapia de primera línea para el tratamiento del GA maculoso y este responde a la misma terapéutica que los otros tipos de GA. Para terminar, queremos destacar el rol del dermatólogo en el diagnóstico de esta patología, que abre las puertas para el tratamiento integral y multidisciplinario del paciente y sus comorbilidades.

maculoso. Dermatol Argent 2016:22:135-139.

12. Nebesio $C L$, Lewis $C$, Chuang TY. Lack of an association between granuloma annulare and type 2 diabetes mellitus. $\mathrm{Br}$ J Dermatol. 2002;146:122-124.

13. Yun JH, Lee JY, Kim MK, Seo YJ, et ál. Clinical and pathological features of generalized granuloma annulare with their correlation: a retrospective multicenter study in Korea. Ann Dermatol. 2009;21:113-119.

14. Piette EW, Rosenbach M. Granuloma annulare: Pathogenesis, disease associations and triggers, and therapeutic options. J Am Acad Dermatol. 2016;75:467-479.

15. Li A, Hogan DJ, Sanusi ID, Smoller BR. Granuloma annulare and malignant neoplasms. Am J Dermatopathol. 2003;25:113-116.

16. Yang CS, Teeple M, Muglia J, Robinson-Bostom L. Inflammatory and glandular skin disease in pregnancy. Clin Dermatol. 2016; 34:335-343.

17. Magalhães GM, Guimarães CF, Paula MC. Case por diagnosis. Patch granuloma annulare. An Bras Dermatol. 2017;92:419-420.

18. Garcia Font M, Curcó Botargues N, Oagerols Bonilla X, Vives Vilá P. Granuloma anular maculoso. Siete nuevos casos. Med Cutan Iber Lat Am. 2004;32:23-26.

19. Trejo Acuña JR, González González M, Figueroa Benítez E, Marín Rivera D. Granuloma anular diseminado. Rev Cent Dermatol Pascua 2019;28: 65-70.

20. Errichetti E, Stinco G. Dermatoscopy of granulomatous disorders. Dermatol Clin. 2018;36:369-375.

21. Levin NA, Patterson JW, Yao LL, Wilson BB. Resolution of patchtype granuloma annulare lesions after biopsy. J Am Acad Dermatol. 2002;46:426-429.

22. Lukács J, Schliemann S, Elsner P. Treatment of generalized granuloma annulare. A systematic review. J Eur Acad Dermatol Venereol 2015;29:1467-1480. 